\title{
Simulation of Water Conservancy Scheduling System Based on an Optimized Dijkstra - Genetic Algorithm
}

\author{
Ming Wang \\ New Media College, Zhejiang university of Media and Communications, \\ Hangzhou, Zhejiang, 310018, China
}

\begin{abstract}
Dynamic optimization scheduling strategy is carried out based on a comprehensive objective function which is constructed for reservoirs. Corresponding constraints and conditions are generally designed, and then the solution of the objective function is worked out based on those constraints and finally an optimal scheduling scheme suivable for reservoirs is acquired in combination with actual conditions. Focusing on the problems above, this study combines Dijkstra algorithm with genetic algerithm (GA) effectively and makes full use of their advantages. Consequenty, an optimized Dijkstra genetic algorithm $(D-G A)$ is obtained and applied in the scheduling scheme. First of all, the thesis preliminarily introduces relevant algorithms on water consewancy project and sets up a data model consistent with the actual situation. Secondly, this work analyzes the two algorithms, improves the Dijkstra algorithm and applies D - GA to solution optimization. Thirdly, this study compares the results obtained by using D-GA and GA respectively and finally completes the simulation of water conservancy scheduling system.

Keywords: Dijkstra - genetic algorithm; Water conservancy scheduling; genetic algorithm; Dijkstra algorithm

\section{Introduction}

Water is a life-sustaining necessity. At present, how to efficiently improve the utilization of water resources [1 hats become a livelihood issue and drawn extensive attention from countries and the society. Generally speaking, the design and construction of water conseryancy engineering depend on natural environment, but in fact, the flow of rivers is uncontrollable 221. Various large and small reservoir nodes [3] are also hubs of the water conservancy project and whether reservoirs can work normally is decided by the runoff volume of the (1)er reach [4]. To date, a reliable method for effective control of the runoff volume has not been found although the existing measures have been taken to monitor the runoff volume of reaches and the rule of data has generally grasped. Water resources $\mathrm{W} 11 \mathrm{~b}$ be in short supply in the following time if the runoff volume of reaches is wrongly grasped and water is drained excessively as originally planned when there is no enough water, thus affecting people's normal life [5]. Accordingly, a large amount of vater has to be drained, causing the waste of water resources, if water is stored over cautously in the month of enough water. Under this circumstance, an effective scheduling scheme is required to reasonably dispatch water resources, so as to use precious water resources in an efficient and rational way [6-7]. This study explores the optimization of water conservancy dispatching and realizes the simulation of water conservancy scheduling system. In the process of optimization, an improved Dijkstra algorithm is used to set the initial population and then genetic algorithm (GA) is adopted for the optimization of solutions in combination with the actual situation.
\end{abstract}




\section{Research Methods}

\subsection{Basic Approach for Water Conservancy Optimal Optimization}

Under normal conditions, water resources can be scheduled periodically. A year is divided into $\mathrm{T}$ periods, $\mathrm{t}=(1,2,3, \ldots \mathrm{T})$. Water conservancy dispatching involves a variety of parameters, including state and decision variables, etc. In general, state variables are uncontrollable, involving water yield $\mathrm{V}$, water volume $\mathrm{Vt}$ at time $\mathrm{t}$ before water storage, water volume $\mathrm{Vt}+1$ at time $\mathrm{t}$ after water storage. Decision variables refers to variables for decision description, which is set as $\mathrm{Dt}$ if the state variable at time $\mathrm{t}$ is $\mathrm{Vt}$, and state variable is changed as $\mathrm{Vt}+1$ after the decision-making.

Therefore, the state of reservoir can be denoted as:

$$
D_{t}: \mathrm{V}_{t} \rightarrow V_{t+1}
$$

Besides, there is a state transfer equation:

$$
V_{t+1}=\mathrm{V}_{t}+Q_{t}-q_{t}
$$

Where Vt refers to the water volume of the reservoir at time $t$ before scheduling; $Q t$ refers to water volume entering the reservoir from upstreams at time $t$ after decision-making; qt refers to water discharge of the reservoir at time $t$.

Some reservoirs are constructed for specific purposes. In this study, maximum generating volume is taken as the ultimate aim and some auxiliary constraint functions are additionally set.

Water levels of the reservoir in flood and non-flood seasonsegre restrained as follows:

In the non-flood season, $\mathrm{d} \leq \mathrm{Ht} \leq \mathrm{n}$, of which, $\mathrm{t}=(1,2,3, \ldots \mathrm{T})$, Ht refers to water level of the reservoir at time $t(d \sim n)$; $d$ refers to minimumyalue of water level; $n$ refers to highest allowed value of water level.

In the flood season, $\mathrm{d} \leq \mathrm{Ht} \leq \mathrm{h}$. Herein, $\mathrm{Ht}$ refers to water level of the reservoir at time $\mathrm{t}$ $(\mathrm{d} \sim \mathrm{h})$; d refers to minimum value of water level (lower limit of water level); h refers to highest allowed value of water level (upper limil of water level).

\subsection{Dijkstra Algorithm}

Dijkstra algorithm as a classicalgorithm is able to calculate the shortest path from any one node to other nodes in a directed digraph of nonnegative weights with the method of breadth first search (BFS), which is a single-source shortest path problem (SSSP) [8].

As to Dijkstra algorithm, $G=(V, E)$ is used to be on behalf of a directed digraph of weights; $\mathrm{S}$ refers to the collection of vertices of the shortest paths. Only one original point $\mathrm{S} 0$ is contained in set $S$ at the initial time point, the shortest path from S0 to other points is added into set $\$$, and the rest of points are expressed as V-S. After continuous processing in this way, a shortest path is obtained and corresponding points are deleted from V-S after being added into $S$. The algorithm comes to an end until all points in the digraph are added into $S$. During the algorithm processing, the shortest distance from the original poin $(S 0$ to all points in $S$ is always ensured to be less than or equal to the distance from the original point $\mathrm{S} 0$ to each point in V-S.

The process of Dijkstra algorithm can be obtained as shown in Figure 1. 


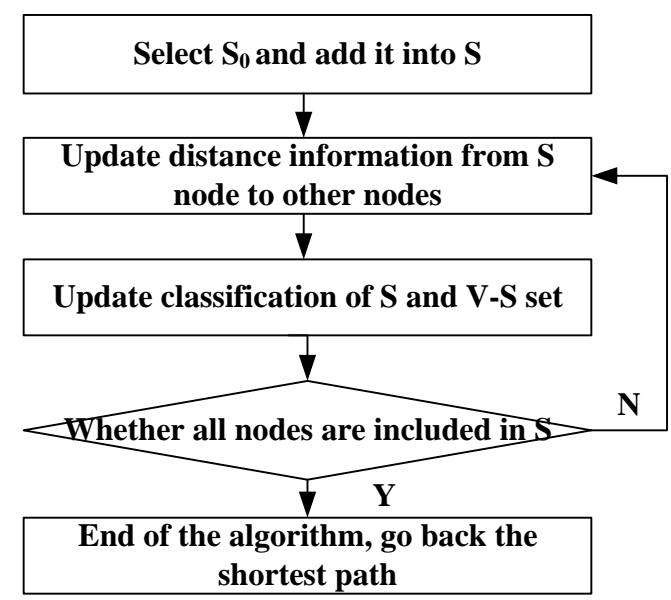

Figure 1. Process of Dijkstra Algorithm

\subsection{Genetic Algorithm}

Genetic algorithm (GA) is a bionics algorithm put forward by an American professor by the name of J. Holland in 1975, which integrates Darwin's blological evolutionism and also reflects the laws of genetics [9]. The specific ontent of GA can be described below: it imitating the biological evolutions in the nature sets up a model for the unsolved problems and regards them as a biological population and then uses some techniques to code populations and confirm the size of initial poputation First of all, a group of individuals is generated randomly ant the fitness of each individual is calculated according to the objective function. The individuak with a good fitness are considered to be optimal. Secondly, the optimal individual in each generation is selected for crossover and mutation (genetic behaviors) On this way, a new generation of individuals which is more adapt to the environment is obtained compared with parental generation. Thirdly, the algorithm stops when certan genetic algebra is set or the difference between the two generations is stipulated to be less than a preset value, and then the latest generation of individuals is acquired, 1.e., the optimal solution [10]. Process of GA is shown in Figure 2.

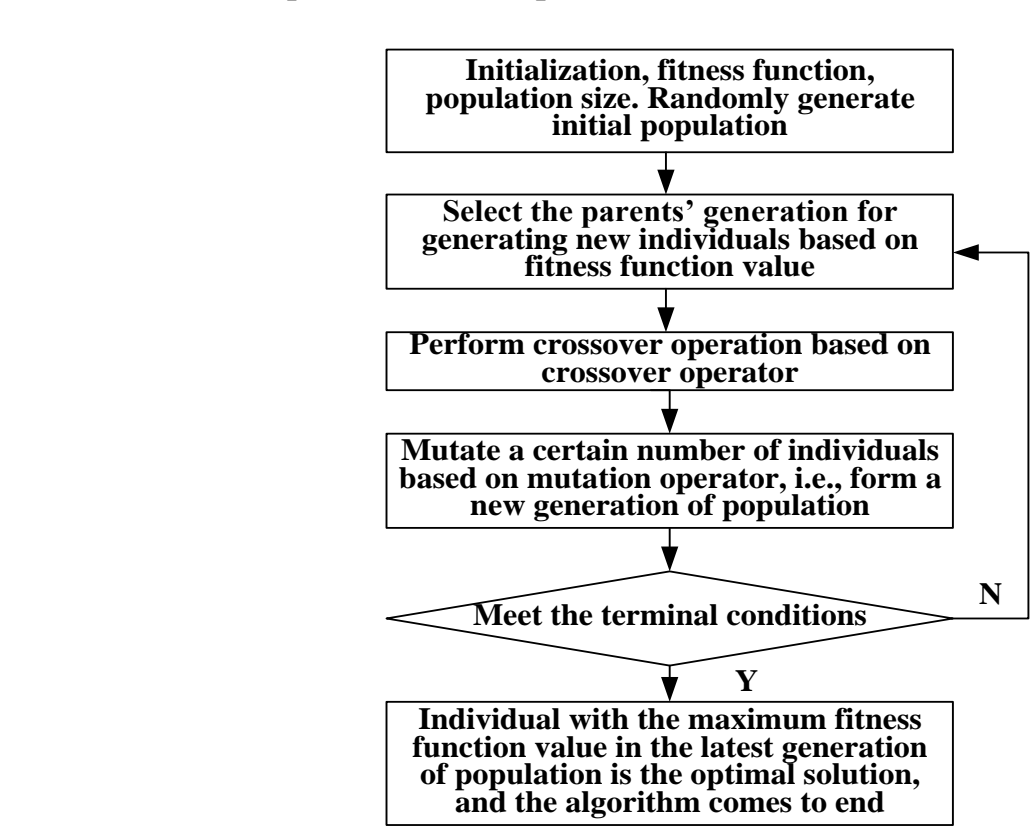

Figure 2. Process of GA 


\section{Application of D-GA Algorithm in Water Conservancy Scheduling System}

\subsection{Improvement and Verification of Dijkstra Algorithm}

Water conservancy scheduling is costly and usually associated with the location of reservoir. Therefore, this study adopts the Dijkstra algorithm which is usually used for processing path problems. This algorithm is improved as follows.

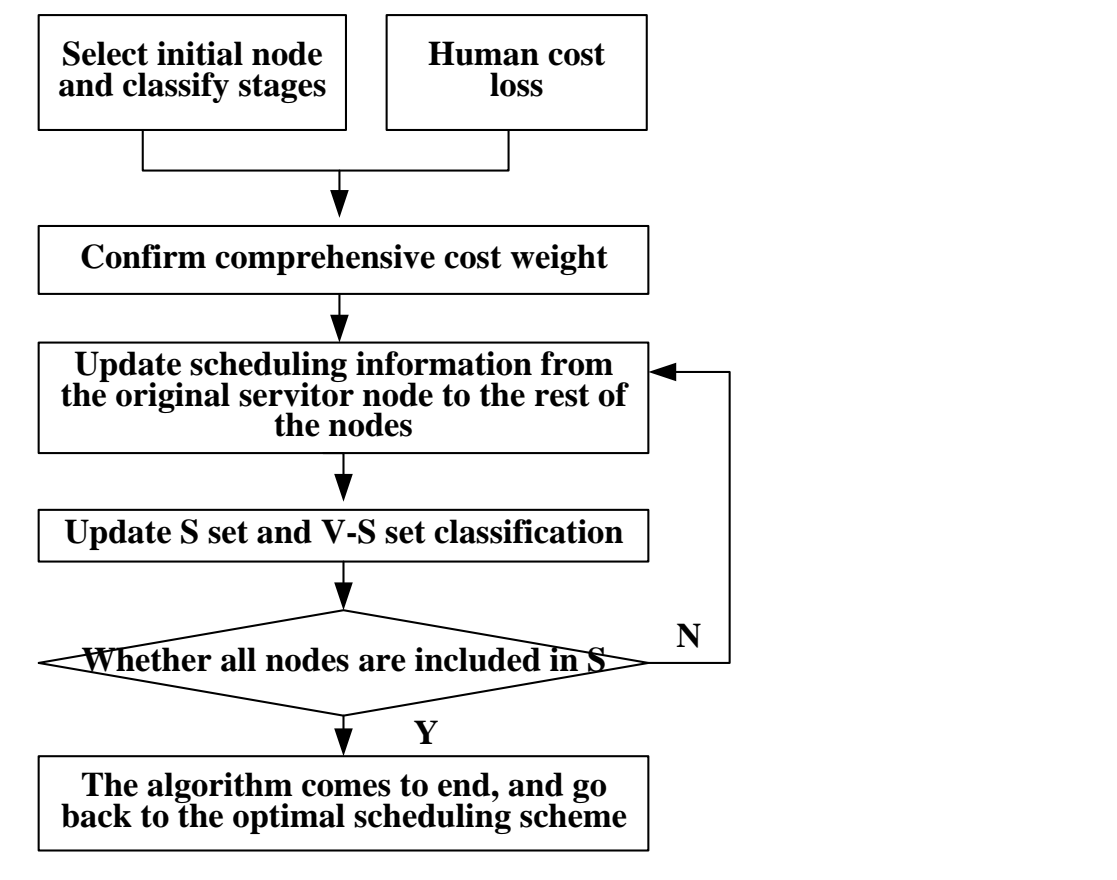

Figure 3. Process of Dijkștra Algorithm Improvement

Normally, the cost of manpower and time from reservoirs A to B is not completely identical with the expense from reseryoirs B to A in the reality. Taking the cost between the two reservolinodes as the average cost of the two places, this study assumes the costs of manpower in reservoirs $\mathrm{A}$ and $\mathrm{B}$ as $\mathrm{C} 1$ and $\mathrm{C} 2$, then the average value $\mathrm{C}$ is figured out:

$$
\text { 2. } C=C_{1}\left(\frac{C_{1}}{C_{2}}+C_{1}\right)+C_{2}\left(\frac{C_{2}}{C_{1}}+C_{2}\right)
$$

In a general way, a variety of factors should be taken into consideration during the confirmation of the final cost in the process of water conservancy scheduling, involving the location of reservoir and the distance between nodes, etc [11]. Assume the distance betweer two reservoirs as S, water wastage of reservoir A and B as W1 and W2, cost of manpower in reservoirs $\mathrm{A}$ and $\mathrm{B}$ as $\mathrm{C} 1$ and $\mathrm{C} 2$ and other losses as L, the total wastage R is finally acquired:

$$
R=S\left(W_{1} C_{1}\left(\frac{C_{1}}{C_{2}}+C_{1}\right)+W_{2} C_{2}\left(\frac{C_{2}}{C_{1}}+C_{2}\right)\right)+L
$$

Hence, the overall cost is obtained according to different locations of reservoirs and human resource expenses in various regions, i.e., the scheduling cost between different reservoirs. 


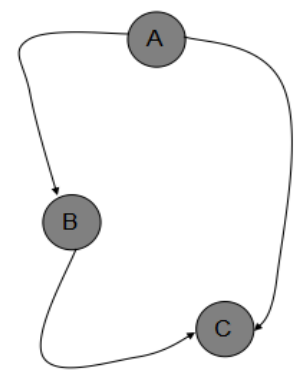

Figure 4. A Simple Second-Level Reservoir

To prove the correctness and superiority of the improved Dijkstra algorithm in managing specific problems, this study takes a simple second-level reservoir scheduling as an example (Figure 4). Besides ensuring small water resource loss, maximizing generating capacity is also considered as the final goal of water scheduling. In view of the scheduling results of various reservoirs, this study compares the results obtained by applying the improved Dijkstra algorithm and the original algorithm (Tables 1,2 and 3)

Table 1. Comparison of Scheduling Results (1)

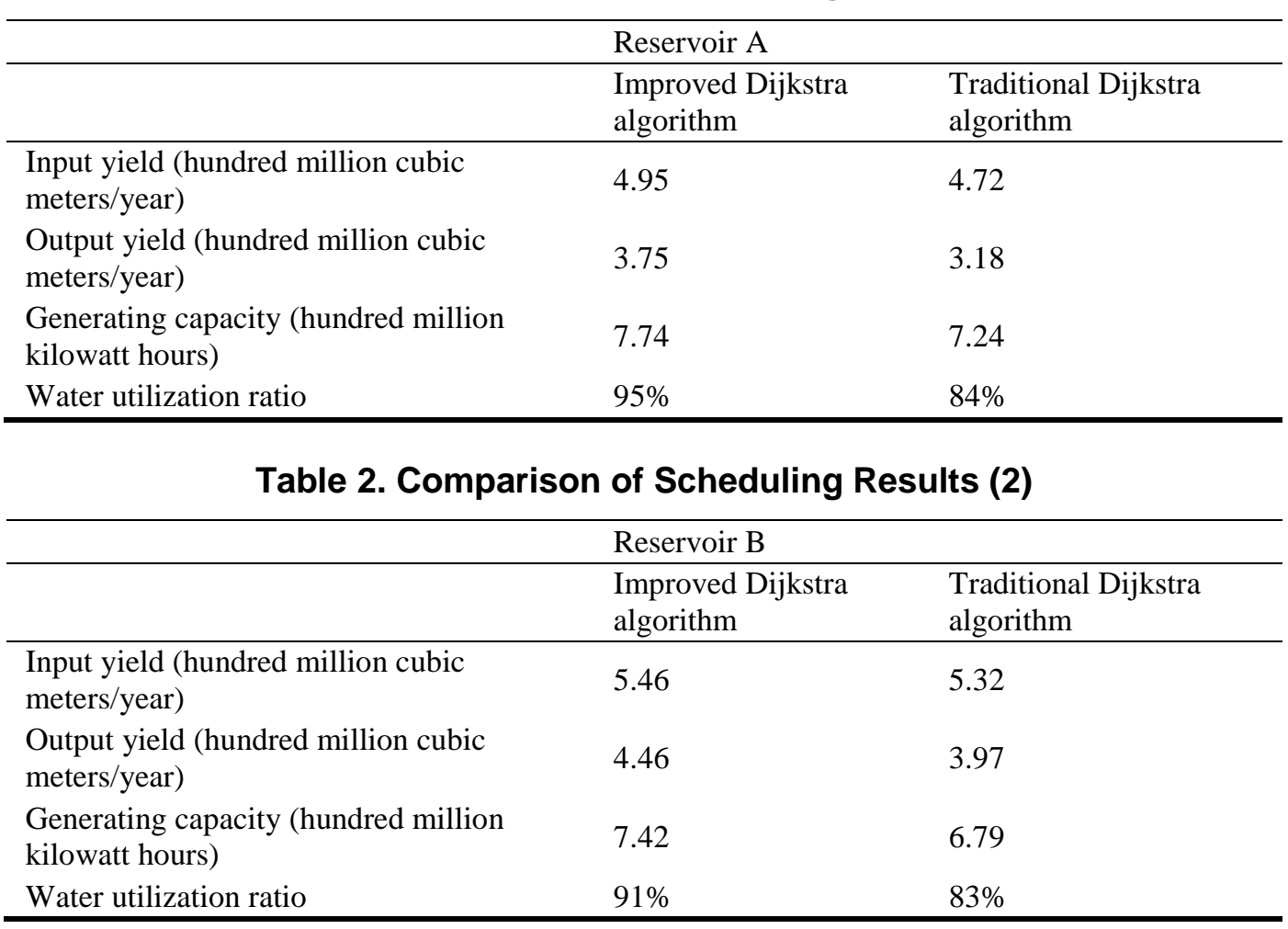


Table 3. Comparison of Scheduling Results (3)

\begin{tabular}{lll}
\hline & Reservoir C & \\
\hline & $\begin{array}{l}\text { Improved Dijkstra } \\
\text { algorithm }\end{array}$ & $\begin{array}{l}\text { Traditional Dijkstra } \\
\text { algorithm }\end{array}$ \\
\hline $\begin{array}{l}\text { Input yield (hundred million cubic } \\
\text { meters/year) }\end{array}$ & 5.65 & 5.31 \\
$\begin{array}{l}\text { Output yield (hundred million cubic } \\
\text { meters/year) }\end{array}$ & 4.72 & 4.26 \\
$\begin{array}{l}\text { Generating capacity (hundred million } \\
\text { kilowatt hours) }\end{array}$ & 8.84 & 8.06 \\
Water utilization ratio & $96 \%$ & $87 \%$ \\
\hline
\end{tabular}

\subsection{Overview of D-GA Algorithm}

The scheduling system is concerned about minimum cost, instead of minimum path. In the process of the applicaiotn of GA, the original GA selects individuals randomly while D-GA algorithm confirms the initial population by using the improved Dijkstra algorithm which gives full play to the advantages of Dijkstra algorithm. Process of the improved GA is displayed in Figure 5.

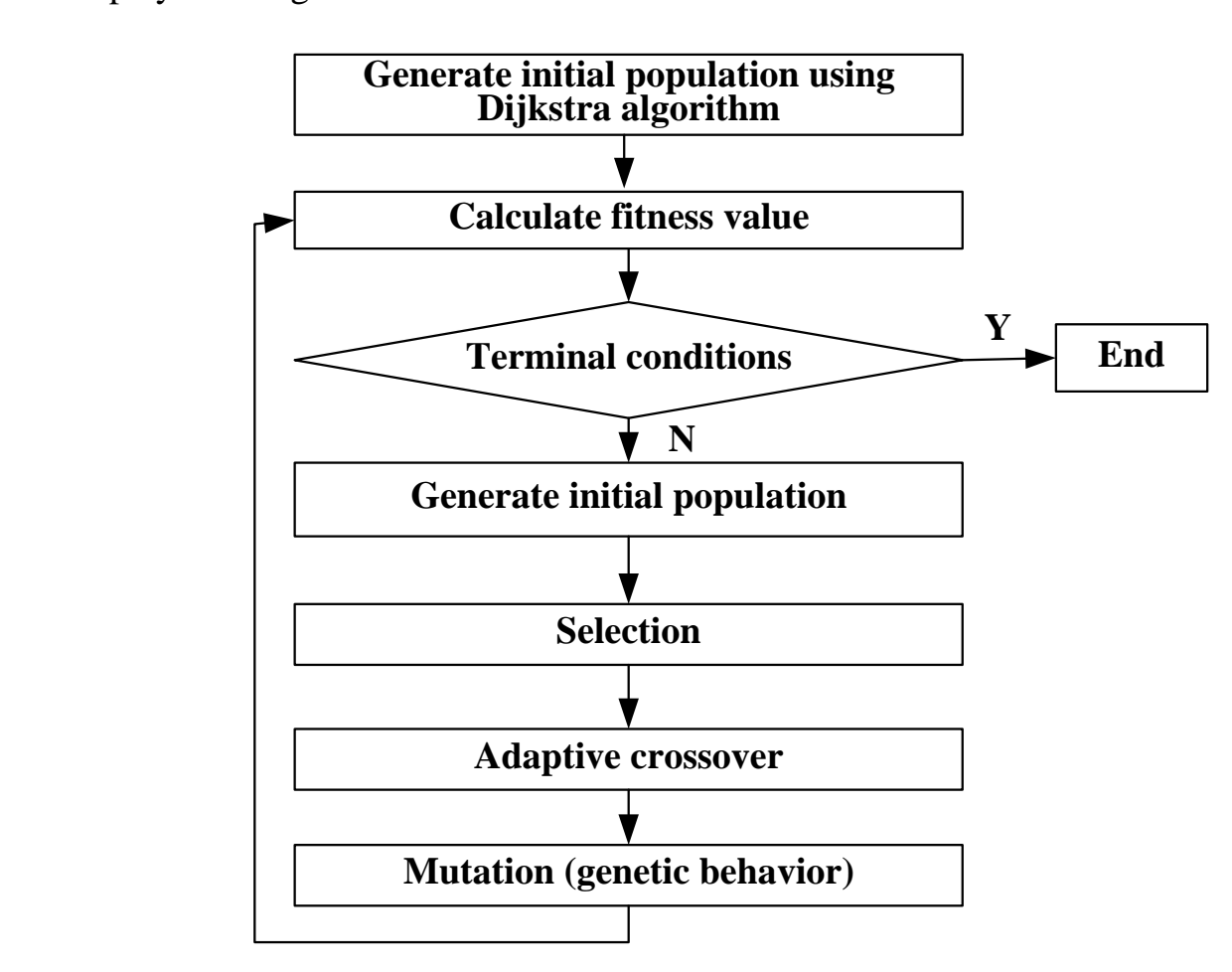

Figure 5. Process of the Improved GA

\subsection{Confirmation of the Initial Population}

A very important work in the application of GA is to confirm the size and content of the initial population. The central idea of Dijkstra algorithm lies in finding out the least-cost path and the next node that completes the shortest path, i.e., the population of minimum cost in water conservancy scheduling. In addition, after the confirmaiton of the gerenration of the initial populaiton, one of the most important problems is how to determine the size of the initial population. The quality of the confimaiton of population 
size directly affects the effectiveness of GA. Too small population size is likely to cause big changes in the process of GA optimization and search termination in the immature stage, thus producing premature convergence. Too large populaiton size can increase calcualtion amount although it ensures the diversity of population, avoids premature convergence and reduces the probability of producing local solution. Hence, an excess of individuals increases the time of calcualting fitness function value and affects the computational efficiency.

\subsection{Confirmation of Fitness Function}

A dynamic optimization scheduling strategy is performed based on a comprehensive objective function which is constructed for reservoirs. Corresponding constraints and conditions are generally designed, and then the solution of the objective function is worked out based on those constraints and finally an optimal scheduling scheme suitable for reservoirs is acquired in combination with actual conditions [12]. Individuals in $a^{f}$ population can be confirmed by a fitness function which is mentioned in GA. In other words, it can figure out which individuals have better adaptability and whichindividuals should be eliminated. The goal of reservoir scheduling in this study is to keep maximum generating capacity of the known reservoir. After analysis, thê objective function can meet the requirements of the fitness function and can be taken as the finess function directly without any revision.

Herein,

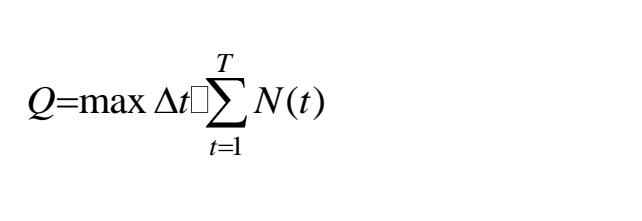

Where $\mathrm{T}$ refers to the total number of eycles $(\mathrm{t}=1,2,3, \ldots \mathrm{T})$. Assume there are $\mathrm{i}$ reservoirs, each reservoir can be regarded as a power station $(\mathrm{i}=1,2,3, \ldots \mathrm{I})$ and every small power station is numbered. Q is used for representing the maximum power generation of the reservoir; $\mathrm{N}(\mathrm{t})$ refêrs to the total power generation of i reservoirs (power stations) within the the cycle and $N(i, t)$ refers to the generating capacity of the ith reservoir in the theycle.

\subsection{Improvement of Crossover Operator}

Crossover operator is an important step in GA. In crossover operation, crossover operator is confirmed first and then part of the individuals of two non-crossed individuals are exchanged according to certain rules, thus obtaining two new individuals [13]. Besides crossover algorithm, crossover modes should also be confirmed during crossover operationand crossover positions of two non-crossed individuals are preset in advance. Thereare a variety of crossover modes and their qualities need to be evaluated before normal crossover operation, so as to select a suitable crossover method for water conservancy scheduling.

(1)Single-point crossover

Single-point crossover, the simplest and most convenient operation method among various crossover modes, refers to selecting two points with the same relative position as crossover points for two individual sequences. Only genes of the two positions are exchanged and two new individuals then come into being. In the actual crossover operation, the location of crossover points can be changeable. A gene sequence has $\mathrm{N}$ genes, so there are $\mathrm{N}$ possibilities for the selection of crossover point.

(2)Two-point crossover

Two-point crossover refers that two individuals participating in crossover operation 
cross at two crossover points and the position of each point crosses at one single point, thus a filial generation is acquired. In the actual crossover operation, the location of two-point crossover is also not fixed. A gene sequence has $\mathrm{N}$ genes, then there are $\mathrm{N}$ $(\mathrm{N}-1) / 2$ kinds of positions.

(3)Multi-point crossover

Multi-point crossover is derived from single-point crossover and two-point crossover. In N-point crossover operation, multi-point crossover degenerates into a single-point crossover if $\mathrm{N}$ is equal to 1 ; another special situation happens when $\mathrm{N}$ is equal to 2, i.e., two-point crossover. In other words, multi-point crossover is a universal crossover behavior. We recommend that $\mathrm{N}$ value should not be too big in an actual genetic operation as too big $\mathrm{N}$ value is easy to result in fast population change and large difference in characteristics of two generations of species although it can ensure the diversity of species, thus an optimal solution cannot be avaliable.

(4)Uniform crossover

Uniform crossover refers to confirming which one is going to cross based on preset shielding information. For example, two non-crossed gene sequences are five and shielding information is 10110 , which indicate that a crossover operation is performed on the 1st, 3rd and 4th genes of the two non-crossed gene sequences?

After comparing various crossover modes above, two-point crossover is adopted in this study, which is simply operated and able to increase the diversity of species as well as gurantee slow evolution of species. Crossover operato is confirmed first. Pre-set values are mostly taken as crossover operators in actual application, which rarely change during the whole genetic operation. Adaptive crossover operatoy is adopted in this study.

Assume the number of individuals in a population as $\mathrm{N}, \operatorname{gmax}$ is used for expressing function value of the individual with maximum fitness in the current population, g0 represents average value of the fitness function value of all individuals in the current population, so $\mathrm{g} 0=(\mathrm{g} 1+\mathrm{g} 2+\ldots+\mathrm{gn}) \mathrm{n}, \mathrm{Pc}$ refers to crossover operator and $\mathrm{g}$ refers to the fitness of the individual with a relatively large fitness function value in two individuals participating in the crossover, so the following eomputational formula is obtained:

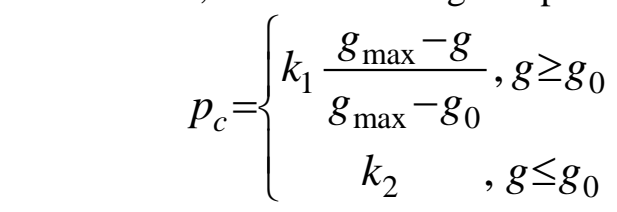

In this way, the value of Crossover operator can be adjusted adaptively in the process of genetic operation so as o guarantee the diversity of species, avoid algorithm stagnation or prematuration and oftingize the performance of the original algorithm.

\subsection{Confirmation of Terminal Conditions}

From the perspective of setup of genetic algorithm, it is very important to design termina conditions. The confirmation of terminal conditions of GA will affect its efficency as well as the quality of results to a large extent. The delicately designed genetic operation is unable to be embodied if termination conditions are set conforming no actual situation.

Therefore, the rate of change of individuals can be obtained and used for determining whether the algorithm should be terminated. In other words, the fitness of population tends to be steady if the rate of change of fitness is lower than a given value $\mathrm{z}$ within $\mathrm{n}$ generations; otherwise, the population changes a lot and is unstable. That is to say, the current population is not an approximately optimal solution and can still be optimized. Besides the two conditions above, there is another special case. However, the rate of change of fitness of population is still not small enough, which means that the population is unstable. At this moment, there is no way to operate it endlessly considering the price and expense of algorithm operation, so the algorithm should be terminated and the 
obtained population is taken as the approximately optimal solution of problem domain.

\section{Design of Water Conservancy System and Model Realization}

\subsection{Design of Functional Diagram of Water Conservancy System}

D-GA algorithm is obtained by improving Dijkstra algorithm and GA and the relevant data of the reservoir are collected. After verification, D-GA algorithm being capable of optimizing complex water conservancy scheduling problems is believed to have obvious advantages over traditional GA.

Firstly, plenty of elements of the reservoir should be considered during the design of water conservancy scheduling system (Figure 6) [14]. Requirements for water level in different periods are diverse [15]. Moreover, the system is supposed to store annual scheduling and water level information, so as to guide work in the future.

Next, demands for water conservancy scheduling system are analyzed and cleared up so as to confirm function module of simulated scheduling system. And finally the system is confirmed to be made up of three stages, including conventional dispatching, dynamic optimal dispatching and manual dispatching [16].

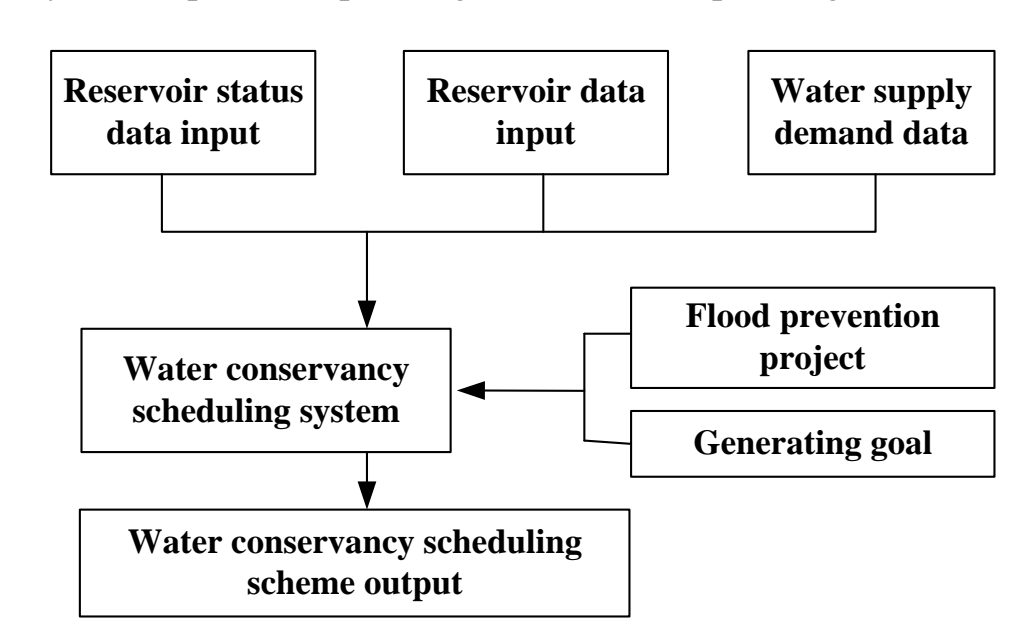

Figure 6. Design o Water Conservancy Scheduling System

\subsection{Instance Analysis}

A reservoir goup is selected and applied to develop a simulated scheduling system. A reservoir group consisting of 5 reservoirs is adopted for verification and their basic irformation is displayed in Tables 4, 5 and 6 below.

Table. 4. Water Supply of the Reservoir Group

\begin{tabular}{llll}
\hline \multirow{2}{*}{ Reservoirs } & \multicolumn{2}{l}{ Water supply (ten thousand cubic meters) } \\
\cline { 2 - 4 } & $\begin{array}{l}\text { Residential water } \\
\text { consumption }\end{array}$ & $\begin{array}{l}\text { Industrial water } \\
\text { consumption }\end{array}$ & $\begin{array}{l}\text { Agricultural } \\
\text { irrigation }\end{array}$ \\
\hline Number 1 & 0.37 & 0.29 & 7.25 \\
Number 2 & 0.39 & 0.35 & 8.42 \\
Number 3 & 0.48 & 0.21 & 9.31 \\
Number 4 & 0.29 & 0.27 & 7.21 \\
Number 5 & 2.02 & 2.54 & 9.19 \\
\hline
\end{tabular}


Table. 5. Water Level Limit of the Reservoir Group

\begin{tabular}{llll}
\hline \multirow{2}{*}{ Reservoir } & \multicolumn{2}{l}{ Water level limit (meter) } \\
\cline { 2 - 4 } & Lower limit & Normal average water level & Upper limit (flood prevention) \\
\hline Number 1 & 91 & 113 & 124 \\
Number 2 & 111 & 121 & 132 \\
Number 3 & 136 & 147 & 136 \\
Number 4 & 95 & 106 & 117 \\
Number 5 & 109 & 122 & 133 \\
\hline
\end{tabular}

Table. 6. Generating Capacity and Reliability of the Reservoir Group

\begin{tabular}{llll}
\hline \multirow{2}{*}{ Reservoir } & \multicolumn{2}{l}{ Water supply (ten thousand cubic meters) } \\
\cline { 2 - 4 } & Generating capacity (ten thousand kilowatt hours) & Reliability \\
\hline Number 1 & 84 & 0.92 \\
Number 2 & 111 & 0.78 \\
Number 3 & 134 & 0.88 \\
Number 4 & 107 & 0.95 \\
Number 5 & 124 & & 0.97 \\
\hline
\end{tabular}

Water conservancy scheduling results of the 5 reservoirs are acquired after system optimization. The responsibility on reservoir number 5 is heavier in water conservancy scheduling. Comparing the obtained scheduling results with the scheduling results of reservoir number 5 in recentyears, the following conclusion can be drawn (Table 7):

Table. 7. Comparison on the Obtained Scheduling Results and the Results in Recent Years

\begin{tabular}{|c|c|c|c|}
\hline Year & $\begin{array}{l}\text { Total power generation } \\
\text { (hundred million kilowatt }\end{array}$ & $\begin{array}{l}\text { Satisfaction degree } \\
\text { of water supply }\end{array}$ & $\begin{array}{l}\text { Times of water } \\
\text { level warning }\end{array}$ \\
\hline 2010 & 2892 & $76.98 \%$ & 16 \\
\hline 2011 & 29.38 & $79.62 \%$ & 14 \\
\hline 2012 & 31.64 & $82.31 \%$ & 15 \\
\hline 2013 & 33.12 & $87.64 \%$ & 13 \\
\hline 2014 & 34.06 & $91.25 \%$ & 16 \\
\hline $\begin{array}{l}\text { Scheduling results } \\
\text { obtained by using the }\end{array}$ & 36.24 & $94.56 \%$ & 12 \\
\hline
\end{tabular}

By analyzing and comparing the results, D-GA algorithm has obvious advantages in optimal dispatching, which can meet the requirements of water conservancy scheduling from the perspective of satisfaction degree of water supply and maximize economic benefits as well. 


\section{Conclusion}

This study first introduces relevant water conservancy scheduling algorithms, summarizes the generation of the concept of water conservancy scheduling as well as its development history, and then analyzes the existing problems in water conservancy scheduling and provides some optimization measures. Moreover, this thesis also constructs a mathematical model, optimizes Dijkstra algorithm combining the advantages of Dijkstra algorithm and GA, then applies D-GA in optimizing scheduling schemes and finally develops a simulated water conservancy scheduling system. Thus, it can be concluded that the improved D-GA is proved to be superior to the original algorithm in optimizing water conservancy scheduling.

\section{Acknowledgments}

2015 Natural Science Foundation of Zhejiang Province, P. R. China: The Research of Portrait generation based on the study of human face attributes. The project No. is LQ15F020005.

\section{References}

[1] N. N. Kourgialas and G. P. Karatzas, "A Modeling Approach for Agricultural Water Management in Citrus Orchards: Cost-effective Irrigation Scheduling and Agrochemical Transport Simulation", Environmental Monitoring and Assessment, vol. 187, no. 7. (2015), pp. 462

[2] L. I. Fei, F. Y. Min. and L. U. J. You, "Problems and Improvements of Water Conservancy Projects Combined with Schistosomiasis Control in River Beaches", Chinese Journal of Schistosomiasis Control, vol. 24, no. 3, (2012), pp. 339-41.

[3] H. G. Jones, "Irrigation Scheduling: Advantages and Pitfalls of Plant-based Methods", Journal of Experimental Botany, vol. 55, no. 407, (2004), pp. 2427-36

[4] W. Conejero, J. J. Alarcón, Y. G. Orellanna, E. Nicolás and A. Torrecillas, "Evaluation of Sap Flow and Trunk Diameter Sensors for Irrigat on Scheduling in Early Maturing Peach Trees", Tree Physiology, vol. 27, no. 12, (2007), pp. 1753-9

[5] M. Nie, Y. Yang, M. Liu, C. Yan H. Shi, W. Dong and J. L. Zhou, "Environmental Estrogens in a Drinking Water Reservoir Area in Shanghai Occurrence", Colloidal Contribution and Risk Assessment, Science of the Total Envirenntent, vol. 487 no 14, (2014), pp. 785-791.

[6] M. R. Hogerheijde, E. A. Bergin, C. Brinch, L. I. Cleeves, J. K. Fogel, G. A. Blake, C. Dominik, D. C. Lis, G. Melnick, D Neufeld, O. Panić, J. C. Pearson, L. Kristensen, U. A. Yildiz and E. F. van Dishoeck, "Detection of the Water Reservoir in a Forming Planetary System", Science, vol. 334, no. 6054, (2011) Pp. 338-40.

[7] C. L. Chang, S. L. Lo and S L. Yu, "The Parameter Optimization in the Inverse Distance Method by Genetic Algorithm for Estimating Precipitation", Environmental Monitoring \& Assessment, vol. 117, no. 1-3, (2006), pp. 145155 .

[8] A. Fortini, M. Dijksta a, M. Schmidt and P. P. Wessels, "Wall-Fluid and Liquid-Gas Interfaces of Model Colloid-Polymer Mixtures by Simulation and Theory", Physical Review E Statistical Nonlinear \& Soft Matter Physics, vol. 71, no. 5, (2005), pp. 122-133.

[9] J. T. Kuo, Y. Y. Wang and W. S. Lung, "A Hybrid Neural-genetic Algorithm for Reservoir Water Quality Management", Water Research, vol. 40, no. 7, (2006), pp. 1367-76.

[10] K. Song L. Li, L. P. Tedesco, S. Li, N. A. Clercin, B. E. Hall, Z. Li and K. Shi, "Hyperspectral Determination of Eutrophication for a Water Supply Source via Genetic Algorithm-partial Least Squares (GA-PLS) Modeling", Science of the Total Environment, vol. 426, no. 2, (2012), pp. 220-232.

111 S. Wang, G. H. Huang and Y. Zhou, "A Fractional-factorial Probabilistic-possibilistic Optimization Framework for Planning Water Resources Management Systems with Multi-level Parametric Interactions", Journal of Environmental Management, vol. 172, (2016), pp. 97-106.

[12] A. Heredia-Langner, W. R. Cannon, K. D. Jarman and K. H. Jarman, "Sequence Optimization as an Alternative to de novo Analysis of Tandem Mass Spectrometry Data", Bioinformatics, vol. 20, no. 14, (2004), pp. 2296-2304.

[13] P. J. García Nieto, J. R. Alonso Fernández, F. J. de Cos Juez, F. Sánchez Lasheras and C. Díaz Muñiz, "Hybrid Modelling based on Support Vector Regression with Genetic Algorithms in Forecasting the Cyanotoxins Presence in the Trasona Reservoir (Northern Spain)”, Environmental Research, vol. 122, no. 2, (2013), pp. 1-10.

[14] H. Li, H. J. He, T. F. Li and Z. K. Zhang, "Microbial Activity and Functional Diversity in Rhizosphere of Cucumber under Different Subsurface Drip Irrigation Scheduling", Chinese Journal of Applied Ecology, vol. 25, no. 8, (2014), pp. 2349-54. 
[15] A. Allam, A. Tawfik, C. Yoshimura and A. Fleifle, "Simulation-based Optimization Framework for Reuse of Agricultural Drainage Water in Irrigation", Journal of Environmental Management, vol. 24, no. 172, (2016), pp. 82-96.

[16] L. Chen, C. H. Tan, S. J. Kao and T. S. Wang, "Improvement of Remote Monitoring on Water Quality in a Subtropical Reservoir by Incorporating Grammatical Evolution with Parallel Genetic Algorithms into Satellite Imagery”, Water Research, vol. 42, no. 1-2, (2008), pp. 296-306.

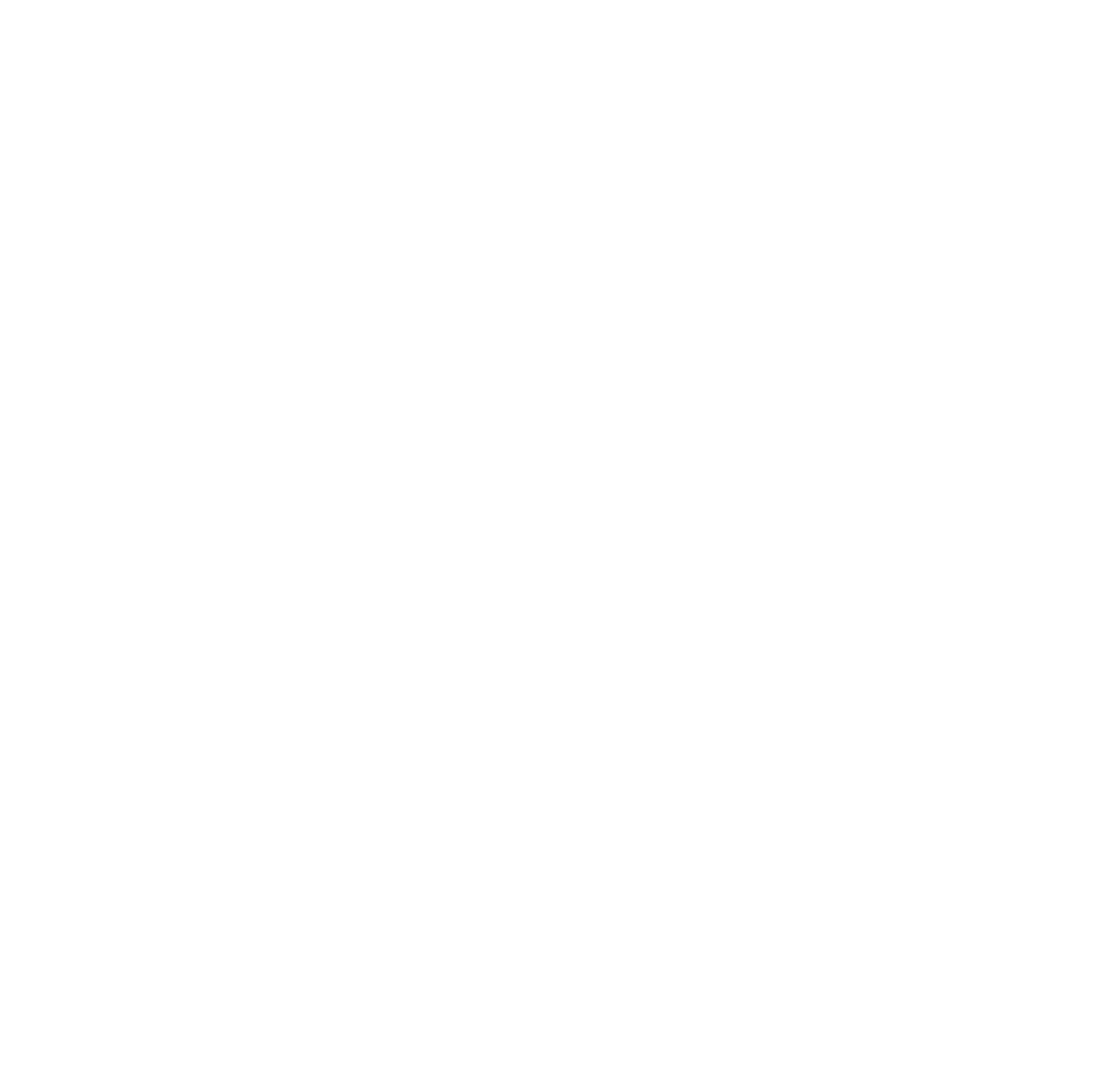

